\title{
Mapping First to Second wave transition of covid19 Indian data via Sigmoid function and prediction of Third wave
}

\author{
Supriya Mondal ${ }^{1}$, Sabyasachi Ghosh ${ }^{2}$
}

July 11, 2021

\footnotetext{
${ }^{1}$ VY Hospital, Adjacent to Kamal Vihar (Sector 12), New Dhamtari Road, Raipur - 492001, Chhattisgarh, India

${ }^{2}$ Indian Institute of Technology Bhilai, GEC Campus, Sejbahar, Raipur - 492015, Chhattisgarh, India
}

\begin{abstract}
Understanding first and second wave of covid19 Indian data along with its few selective states, we have realized a transition between two Sigmoid pattern with twice larger growth parameter and maximum values of cumulative data. As a result of those transition, time duration of second wave shrink to half of that first wave with four times larger peak values. It is really interesting that the facts can be easily understood by simple algebraic expressions of Sigmoid function. After understanding the crossing zone between first and second wave curves, a third wave Sigmoid pattern is guessed.
\end{abstract}

\section{Introduction}

Spreading of the novel coronavirus, covid19, from China to entire globe become so alarming that the World Health Organization (WHO) declared it as a pandemic disease on 11th March 2020 [1, 2]. The data of covid infected, recovered and death are maintained by different countries in their government based websites. Ref. [3] is citing the corresponding website for India. From 2020 to now, a huge amount of works are attempted to fit the covid infected daily cases and cumulative data for predicting the their pattern. Few of them are cited in Refs. $[4,5,6,7,8,9,10,11,12,13,14,15,16,17,18$, $19,20,21,22,23,24,25,26,27,28,29]$, where few Refs. [18, 24] work on second wave data and third wave prediction. Most of the studies are based on SIR model [30, 31, 32] and its extensions. This framework is widely used for other countries, e.g. Ref. [33]. However, a simple logistic function description like Sigmoid function $[34,35,36]$ can also be an easy-dealing tools to understand the epidemic outbreak. In our earlier works $[4,5,6]$, we have used this Sigmoid function framework for predicting first wave of India data. This framework is also used for understanding epidemic size of other countries, for example Refs. [35, 36, 37].

Present work is aimed to explain the existing first and second wave covid19 infection data with the help of two different Sigmoid functions and analyzing their trends, we have predicted third wave pattern. The article is organized as follows. Starting with brief formalism part of Sigmoid function, 
we have discussed about the steps of generating curves in the Sec. (2). Next, in results section, we have described the first and second wave curves including our third wave predicted curves. After analyzing those results, at the end we summarized our work.

\section{Mathematical Framework}

In this framework part, we will discuss quickly about the Sigmoid function which will be used to interpret covid 19 data. Then we will discuss about the steps, through which we proceed.

The form of Sigmoid function is

$$
N(t)=N_{0} e^{\lambda t} /\left(\frac{N_{0} e^{\lambda t}}{N_{m}}+1\right)
$$

where $N_{0}$ is initial number of cases, $\lambda$ is growth parameter, $N_{m}$ is the maximum values, where cumulative case $N(t)$ will be saturated. Here t represents number of days. Now, the time derivative of Sigmoid function is

$$
\frac{d N}{d t}=\lambda N_{0} e^{\lambda t} /\left(\frac{N_{o} e^{\lambda t}}{N_{m}}+1\right)^{2},
$$

which is the number of new cases per day as we see in covid 19 data. Sigmoid function shows exponential behaviour in low values of $t$ but it will saturate to a maximum values $\left(N_{\max }\right)$ at high values of $t$. When we analyze its derivative or slope, then we will get first increasing and then decreasing trends after showing a peak. The peak structure of daily cases depends on three parameters $N_{m}$, $N_{0}, \lambda$. The peak time $t_{p}$, when daily cases reach its highest value, can be expressed as

$$
t_{p}=\frac{\ln \left(N_{m} / N_{0}\right)}{\lambda} .
$$

At $t=t_{p}$ daily cases and cumulative cases are respectively

$$
\left(\frac{d N}{d t}\right)_{p}=\frac{\lambda N_{m}}{4} .
$$

and

$$
N_{p}=N_{m} / 2
$$

Above simple formalism can be useful to describe covid 19 data pattern. In India we found two waves whose cumulative and daily cases data patterns can expressed in terms of two consecutive Sigmoid functions and their time derivatives.

From the first wave of Covid 19 Indian data [3] we find out the values of $t_{p 1},(d N / d t)_{p 1}$ and $N_{m 1}$. These values are used in Eqs. (3) and (4) to find out the values of $\lambda_{1}$ and $N_{01}$. Subscript 1 is added in the notations of different parameters to assign first wave case. For India and some selective states - 1) Maharashtra (MH), 2) Kerala (KL), 3) Karnataka (KA), 4) Tamil Nadu(TN), 5) Andhrapradesh (AP), 6) West Bengal(WB), those parameters are tabulated in Table. (1).

Next, when we will go for corresponding second wave data, we will not get $N_{m 2}$ as it is not till finished and we can not see the second saturated cumulative values. However, we can see the $N_{p 2}$ values from data and by using Eq. (5), we can guess $N_{m 2}$ by making twice of $N_{p 2}$. Here, subscript 2 is 


\begin{tabular}{c|c|c|c|c|c}
\hline State & $\lambda_{1}$ & $N_{m 1}$ & $N_{01}$ & $t_{p 1}$ & $\left(\frac{d N}{d t}\right)_{p 1}$ \\
\hline MH & 0.05 & $19 \times 10^{5}$ & 142 & 190 & $25 \times 10^{3}$ \\
KL & 0.027 & $10 \times 10^{5}$ & 549 & 278 & $9 \times 10^{3}$ \\
KA & 0.048 & $8.6 \times 10^{5}$ & 37 & 209 & $10 \times 10^{3}$ \\
TN & 0.035 & $8 \times 10^{5}$ & 1469 & 148 & $7 \times 10^{3}$ \\
AP & 0.04 & $8.8 \times 10^{5}$ & 606 & 182 & $10 \times 10^{3}$ \\
WB & 0.029 & $5.5 \times 10^{5}$ & 522 & 240 & $4 \times 10^{3}$ \\
\hline India & 0.045 & $94 \times 10^{5}$ & 1000 & 192 & $94 \times 10^{3}$ \\
\hline
\end{tabular}

Table 1: Different parameters of Sigmoid functions, which can grossly describe covid19 first wave data of India and selective states - MH, KL, KA, TN, AP, TN.

added in the notations of different parameters to assign second wave case. Another important point for cumulative data of second wave is that we will redefine it by subtracting first wave maximum values $N_{m 1}$. It means that Eqs. (1) and (2) for second waves will be

$$
N_{2}(t)=N_{02} e^{\lambda_{2} t} /\left(\frac{N_{02} e^{\lambda_{2} t}}{N_{m 2}}+1\right)
$$

and

$$
\frac{d N_{2}}{d t}=\lambda_{2} N_{02} e^{\lambda_{2} t} /\left(\frac{N_{02} e^{\lambda_{2} t}}{N_{m 2}}+1\right)^{2},
$$

where $N_{m 2}=\left(N_{p 2}-N_{m 1}\right) \times 2$. So knowing $N_{m 1}, N_{p 2}$ from data, we can guess about $N_{m 2}$. Although, we should keep in mind that $N_{m 1}+N_{m 2}$ is actual saturated values of second wave case, when we compare it with actual data. The parameters of second waves for India and the selective states are given in Table. (2).

\begin{tabular}{c|c|c|c|c|c|c}
\hline State & $\lambda_{2}$ & $N_{m 2}$ & $N_{02}$ & $t_{p 2}$ & $\left(\frac{d N}{d t}\right)_{p 2}$ & $N_{p 2}+N_{m 1}$ \\
\hline MH & 0.05 & $46 \times 10^{5}$ & 350 & 84 & $67 \times 10^{3}$ & $42 \times 10^{5}$ \\
KL & 0.078 & $20 \times 10^{5}$ & 648 & 103 & $39 \times 10^{3}$ & $20 \times 0^{5}$ \\
KA & 0.11 & $16.8 \times 0^{5}$ & 43 & 96 & $49 \times 10^{3}$ & $17 \times 10^{5}$ \\
TN & 0.085 & $16 \times 10^{5}$ & 151 & 109 & $34 \times 10^{3}$ & $16 \times 10^{5}$ \\
AP & 0.09 & $10 \times 10^{5}$ & 74 & 106 & $24 \times 10^{3}$ & $14 \times 10^{5}$ \\
WB & 0.08 & $9 \times 10^{5}$ & 257 & 102 & $20 \times 10^{3}$ & $10 \times 10^{5}$ \\
\hline India & 0.08 & $2 \times 10^{7}$ & 8579 & 96 & $4 \times 10^{5}$ & $2 \times 10^{5}$ \\
\hline
\end{tabular}

Table 2: Same as Table (1) for second wave.

\section{Results}

We have described the steps, through which we have find the parameters of first and second wave of covid 19 spreading. Fig. (1) shows the Sigmoid function nature of cumulative $N$ and daily case $d N / d t$ 

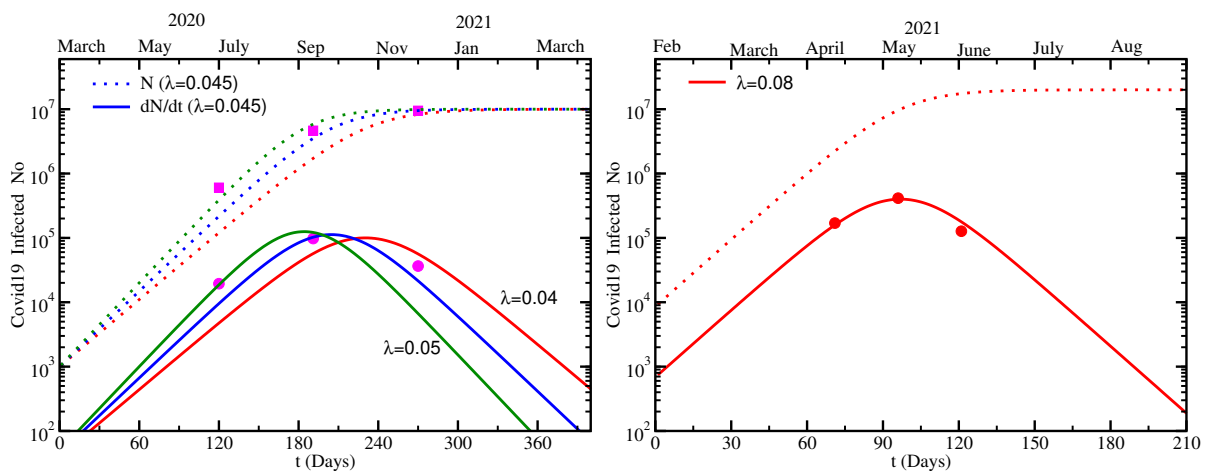

Figure 1: Left panel: Cumulative(squares) and daily cases (circles) data, fitted in Sigmoid curves(dotted line) and their derivatives (solid lines) for first wave. Right panel: Same for second wave.

data of India. In the left and right panels of Fig. (1) represents the data points (squares and circles) and corresponding Sigmoid fitted curves (dotted and solid lines) in first and second waves respectively . We consider 3 main data points of daily and cumulative cases at $t=\left(t_{p}-2 / \lambda\right), t_{p},\left(t_{p}+2 / \lambda\right)$, within which spreading become most dominant. We have taken three different values of $\lambda=0.04,0.045$ and 0.05 to fit the three data points of first wave. In another aspect, Second wave is well fitted with one $\lambda=0.08$. First waves is saturated in $N_{m 1}=10^{7}$ and second waves is saturated in $N_{m 2}=2 \times 10^{7}$ which are already seen in covid 19 data. They are implemented as important inputs to build corresponding Sigmoid curves.

Next, we will generate similar graphs in Figs. (2) and (3) for 6 selective states - MH, AP, WB, TN, KL and KA.In Fig. (2) we noticed that the daily cases data of MH, AP and TN in first wave are well fitted by (time derivative of) Sigmoid function but the same for WB, KA and KL are not so well fitted by Sigmoid function. On the other hand in second wave all those data are favouring the Sigmoid function, which can be seen in Fig. (3). In first wave there was no sharp peak for few states where as peak was clearly seen during second wave almost in every states. This is most probably because of rapidly growing of daily cases in second wave which was lacking for few states in first wave.

In first wave, we find the range of growth parameter $\lambda_{1}=0.03-0.05$ and peak value $\left(\frac{d N}{d t}\right)_{p 1}=$ $(0.04-0.25) \times 10^{5}$. The state level range of growth parameter is quite close to the range of $\lambda_{1}=0.04-$ 0.05 for entire country. Being added of state level peak values, we find $\left(\frac{d N}{d t}\right)_{p 1} \approx 1 \times 10^{5}$ for India. Different states shown peak value at different time points $t_{p 1}$ which are in the range of $t_{p 1}=5-9$ months. India data shows the peak value around $t_{p 1}=6.5$ months. If we analyze second wave then state level ranges are $\lambda_{2}=0.078-0.1$ (excluding $\left.\mathrm{MH} \lambda_{2}=0.05\right),\left(\frac{d N}{d t}\right)_{p 2}=(0.2-0.67) \times 10^{5}$, $t_{p 2}=2.8-3.5$ months and India data shows at $\lambda_{2}=0.08,\left(\frac{d N}{d t}\right)_{p 2} \approx 4 \times 10^{5}, t_{p 2}=3.2$ months. If we compare first and second wave data of India and its different states, then we can notice their ratios as $\lambda_{2} / \lambda_{1} \approx 2, t_{p 2} / t_{p 1} \approx 1 / 2, N_{m 2} / N_{m 1} \approx 2$. Although ratio between peak values of two waves for different states are not quite stable. As example, it is approximately 4 for India, 5 for 



Figure 2: Sigmoid curves(dotted lines) and their derivatives (solid lines) for first wave in MH, AP, WB, TN, KL, KA.


Figure 3: Same as Fig. (2) for second wave.

WB, TN and 13 for KL etc. Considering India data as collective effect, we may conclude that first to second wave transition was just transition of parameters $\lambda_{1} \rightarrow \lambda_{2}=2 \lambda_{1}, t_{p 1} \rightarrow t_{p 2}=t_{p 1} / 2$, $N_{m 1} \rightarrow N_{m 2}=2 N_{m 1}$ and $\left(\frac{d N}{d t}\right)_{p 1} \rightarrow\left(\frac{d N}{d t}\right)_{p 2}=4\left(\frac{d N}{d t}\right)_{p 1}$.

Now let us come to the question whether we can identify the reason for occurring second wave after the first wave? Single answer of this question is really difficult but mutation of virus might be considered as a dominating point. Here, we will try to understand graphically but reader should considered that quantitative message with a very flexible way. In Fig. (4), we have drawn first and second wave daily cases curves in one portrait and we can see an overlapping/crossing zone of them around February, 2021. This is also observed in data (circles) as one can notice that second wave rising is started after Feb, 2021. We have put few selective daily cases data at $t_{p 1}-\frac{2}{\lambda_{1}}, t_{p 1}, t_{p 1}+\frac{2}{\lambda_{1}}$, $t_{p 1}+\frac{4}{\lambda_{1}}, t_{p 1}+\frac{6}{\lambda_{1}}, t_{p 2}-\frac{6}{\lambda_{2}}, t_{p 2}-\frac{4}{\lambda_{2}}, t_{p 2}-\frac{2}{\lambda_{2}}, t_{p 2}, t_{p 2}+\frac{2}{\lambda_{2}}$. One can notice that our Sigmoid curves for first and second waves are well fitted within $t_{p 1, p 2} \pm \frac{2}{\lambda_{1,2}}$. Outside those zones, the trends of data points and curves are going similar although bit of differences in numerical strengths are noticed. Now, assuming mutated virus as a dominating cause of second wave, let us try to describe the data 




Figure 4: Log scale version of left panel of Fig. (5). Here their superposition are presented by brown and yellow solid lines and starting points of second and third wave are marked by red arrows.

as follows. After entering covid19 virus into India from March, 2020, first wave has received its peak around Sep, 2020 and then it went down until Feb, 2021. This imported covid19 virus are started to be mutated and among the different variant, delta virus [38] become more contagious than previous. We may consider this delta virus as a dominating reason for second wave, which is appeared to be started from Feb, 2021 and received peak around first week of May, 2021. Interestingly, first confirmed case of delta virus in India is observed around Oct, 2020, [39] which is quite earlier than Feb, 2021, from when second wave seems to grow. So it is quite interesting fact that the growing pattern of delta virus was hidden from Oct, 2020 to Feb, 2021. We can only see the decay pattern of first wave curve. If we extend our second wave Sigmoid curve before Feb, 2021, then we get $N_{0} \approx 1$ around Oct, 2020. Recovering this empirical point by the simple logistic function is really very interesting fact. So if we crudely assign our first and second wave as the outcome of covid19 virus and mutated/delta virus spreading, then we can find recognize their overlapping zone.

After realizing the transition from first to second wave, we can use the idea for predicting third wave. After mutation of delta virus, recently delta plus $(\delta+)$ virus is first reported in India around June, 2021. It is probably $1.5-2$ times contagious $[40,41]$ than delta virus, although the fact is not very settled [42]. We already noticed that by simply changing $\lambda_{1}=0.04$ and $N_{m 1}=1 \times 10^{7}$ to $\lambda_{2}=0.08$ and $N_{m 2}=2 \times 10^{7}$, we get first to second wave transition. Doubling of the growth parameter can be connected with the two times contagious of delta virus with respect to former covid19 virus. So assuming $\delta+$ virus as two times contagious than $\delta$ virus and assigning $\delta+$ as a dominating cause for third wave, we have drawn another Sigmoid function with $\lambda_{3}=2 \lambda_{2}=0.16$ 



Figure 5: Left: first, second and third wave cumulative (dotted line) and daily (solid line) case Sigmoid curves and few selective data points (circles) of daily cases. Right: Predicted third wave Sigmoid functions - cumulative (dotted line) and daily (solid line) cases for different guess values of growth parameters $\lambda_{3}$ and maximum cumulative value $N_{m 3}$.

and $N_{m 3}=2 N_{m 2}=4 \times 10^{7}$ in Fig. (4). We have started it from June with $N_{0}=1$. As $\delta$ virus started to spread from Oct, 2020 but its actual growing become noticeable from Feb, 2021, similarly $\delta+$ virus started in June, 2021 but its actual growing might be found from mid-Aug, 2021. Most fearing point is that it might go with rapidly blowing pattern. By transforming log scale to linear scale, Fig. (4) is redrawn in left panel of Fig. (5), from where reader can guess the fearing point. Including our predicted third wave curve along with the observed first and second wave curves, we may present them as transitions among three Sigmoid functions, whose parameter transformation can be expressed in a single equation:

$$
\begin{array}{rll}
\lambda_{1} \approx 0.04 & \rightarrow \lambda_{2} \approx 0.08 & \rightarrow \lambda_{3} \approx 0.16 \\
N_{m 1} \approx 1 \times 10^{7} & \rightarrow N_{m 2} \approx 2 \times 10^{7} & \rightarrow N_{m 3} \approx 4 \times 10^{7} \\
t_{p 1} \approx 192 \text { Days } & \rightarrow t_{p 2} \approx 96 \text { Days } & \rightarrow t_{p 3} \approx 48 \text { Days } \\
\left(\frac{d N}{d t}\right)_{p 1} \approx 1 \times 10^{5} & \rightarrow\left(\frac{d N}{d t}\right)_{p 2} \approx 4 \times 10^{5} \rightarrow\left(\frac{d N}{d t}\right)_{p 3} \approx 16 \times 10^{5} .
\end{array}
$$

Since there are no empirical data points from where we can guess third wave parameters, so as a possible numerical range, right panel of Fig. (5) is dedicated for that. By taking $N_{m 3}=(2,4,6) \times 10^{7}$ and $\lambda_{3}=0.12,0.16,6$ curves are plotted and we find that the peak values can be varying from $5 \times 10^{5}$ to $23 \times 10^{5}$ and peak position can be varying from Sep, 2021 to Nov, 2021 .

\section{Summary}

In summary, present work is intended to explain the existing first and second wave covid19 infection data with the help of simple logistic function, called Sigmoid function. From the data points of peak values and peak positions for daily cases of India and its selective states MH, KL, KA, TN, WB, AP, 
we have found the required input parameters of the Sigmoid functions. Our results grossly indicates a transition between two Sigmoid pattern with twice larger growth parameter and maximum values of cumulative data during first to second wave transition. In parallel, time duration of second wave shrink to half of that first wave and peak values of daily cases becomes four times larger. From the basic properties of Sigmoid functions, those changes can be easily realized.

Another interesting aspect is revealed for mutated delta virus observation. Via backward extensions of second wave Sigmoid curve, we can reach to the initial point (Oct, 2020), when delta virus was first observed. Implementing that idea, we can consider June, 2021 as third wave initial point, since next version mutated delta plus virus is observed on that time. Assuming two times contagious nature delta plus than delta virus, we have considered third wave Sigmoid function with doubled growth parameter with respect to second wave Sigmoid functions. Including our third wave prediction, we have sketched three waves in terms of three Sigmoid functions. Their growth parameters and maximum values of cumulative cases are jumping two times than previous; their daily cases peak values are jumping four time; and their epidemic duration shrinks from 12 months to 6 months to 3 months.

\section{References}

[1] WHO, Novel coronavirus (2019-nCoV) situation report - 11 (2020).

[2] WHO, Coronavirus disease 2019 (COVID-19) situation report - 51 (2020).

[3] Covid19 India

[4] S. Mondal, S. Ghosh, Fear of exponential growth in Covid19 data of India and future sketching International Journal of Creative Research Thoughts Vol. 8, pp. 2422 (2020) DOI: 10.1101/2020.04.09.20058933

[5] S. Mondal, S. Ghosh, Possibilities of exponential or Sigmoid growth of Covid19 data in different states of India Indian Journal of Applied Research Vol. 10, issue 6 (2020) DOI: 10.1101/2020.04.10.20060442

[6] S. Mondal, S. Ghosh, Searching the Sigmoid-type trend in Lock down Period covid19 Data of India and its Different States, Journal of Clinical/Pharmaco-Epidemiology Research Vol. 2, issue 2 (2020) DOI: 10.1101/2020.04.10.20060442

[7] A. Bhattacharyya, D. Bhattacharyya, J. Mukherjee, The connection of growth and medication of covid-19 affected people after 30 days of lock down in India International Journal of Scientific Research, vol. 9, issue 7 (2020), DOI : 10.36106/ijsr , DOI: 10.1101/2020.05.21.2010794

[8] A. Bhattacharyya, D. Bhowmik, J. Mukherjee, Forecast and interpretation of daily affected people during 21 days lockdown due to covid19 pandamic in India Indian Journal of Applied Research, vol. 10, issue 5 (2020), DOI : 10.36106/ijar , DOI: 10.1101/2020.04.22.20075572

[9] R. Bag M. Ghosh, B. Biswas, M. Chatterjee Understanding the spatio-temporal pattern ofCOVID-19 outbreak in India using GIS and India'sresponse in managing the pandemic Regional Science Policy \& Practice (2020), DOI: 10.1111/rsp3.12359 
[10] B.S.K. Meghana, V. Kakulapati, State-wise prevalence of covid 19 in India by machine learning approach International Journal of Pharmaceutical Research, vol. 12, Issue 2 (2020), DOI:10.31838/ijpr/2020.SP2.295

[11] A.A.Tiwari, Temporal analysis of covid19 peak outbreak DOI:10.1101/2020.09.11.20192229

[12] A.kotwal et.al., Predictive models of COVID19 in India: A rapid review Medical Journal Armed Forces India, vol. 76, Issue 4, PP 377-386 (2020) DOI:10.1016j.mjafi.2020.06.001

[13] P. Chauhan, A. Kumar, P. Jamdagni, Regression analysis of COVID 19 Spread in India and its different states DOI:10.1101/2020.05.29.20117069

[14] M. Jakhar , P.K. Ahluawalia, A A .Kumar COVID 19 Epidemic Forecast in Different States od India using SIR Model DOI:10.1101/2020.05.14.20101725

[15] D Biswas, S Roy Analyzing COVID-10 Pandemic with a new growth model for population ecology DOI:10.13140/RG.2.2.34847.92324/1

[16] A Rajesh,H Pai, V Roy, S Samonta, S Ghosh, Covid 19 Prediction for India from the existing data and $\operatorname{SIR}(D)$ model study, DOI:10.1101/2020.05.05.20085902

[17] A. Adhikari, A. Pal, A six compartments with time-delay modelSHIQRD for the COVID-19 pandemic in India:During lockdown and beyond, accepted in Alexandria Engineering Journal.

[18] C. Kavitha, A. Gowrisankar 1 , Santo Banerjee, The second and third waves in India: when will the pandemic be culminated? Eur.Phys.J.Plus 136, 596 (2021).

[19] A. Gowrisankar, R. Lamberto, S. Banerjee, Can India develop herd immunity against COVID19?, Eur.Phys.J.Plus 135, 526 (2020) 15.

[20] D. Rafiq, S.A. Suhail, M.A. Bazaz, Evaluation and prediction of COVID-19 in India: A case study of worst hit states. Chaos Solitons Fract. 139, 110014 (2020) 16.

[21] C. Pai, A. Bhaskar, V. Rawoot, Investigating the dynamics of COVID-19 pandemic in India under lock-down. Chaos Solitons Fract. 138, 109988 (2020) 17.

[22] P. Arora, H. Kumar, B.K. Panigrahi, Prediction and analysis of COVID-19 positive cases using deep learning models: A descriptive case study of India., Chaos, Solitons Fract. 139, 110017 (2020) 18.

[23] B.K. Sahoo, B.K. Sapra, A data driven epidemic model to analyse the lockdown effect and predict thecourse of COVID-19 progress in India. Chaos Solitons Fract. 139, 110034 (2020) 19.

[24] R. Ranjan Aryan, S. Mahendra, K. Verma, Characterization of the Second Wave of COVID-19 in India, Doi:https://doi.org/10.1101/2021.04.17.2125566522.

[25] R. Ranjan, Temporal dynamics of COVID-19 outbreak and future projections: A data-driven approach, Transactions of the Indian National Academy of Engineering, pages 1-7, 2020.

[26] R. Ranjan. Covid-19 spread in india: Dynamics, modeling, and future projections, Journal of Indian Statistical Association, 58(2):47-65, 2020. 
[27] A. Padhi et al., Studying the effect of lockdown using epidemiological modelling of COVID-19 and a quantum computational approach using the Ising spin interaction Scientific Reports, 10 (2020) 21741.

[28] S. Roy, An algebraic interpretation of the spread of COVID-19 in India and an assessment of the impact of social distancing World Journal of Advanced Research and Reviews, 06 (2020) 245, DOI: 10.30574/wjarr

[29] D.K. Bagal, A. Rath, A. Barua, D. Patnaik, Estimating the parameters of susceptible-infectedrecovered model of COVID-19 cases in India during lockdown periods, Chaos Solitons \& Fractals, 140 (2020) 110154.

[30] W. O. Kermack and A. G McKendrick, A contribution tothe mathematical theory of epidemics, Proceedings of the royal society oflondon. Series A, Containing papers of a mathematical and physical character, 115 (772):700721, 1927.

[31] H.W. Hethcote, The mathematics of infectious diseases, SIAM review, 42 (4) :599653, 2000.

[32] R.M. Anderson, B. Anderson, and R.M. May, Infectious diseases ofhumans: dynamics and control, Oxford university press, 1992.

[33] N. Hussain, B. Li, Using R-studio to examine the COVID-19 Patients in PakistanImplementation of SIR Model on Cases International Journal of Scientific Research in Multidisciplinary Studies, Vol.6, Issue.8, pp.54 (2020)

[34] Wikipedia : Sigmoid function

[35] M. Batista, Estimation of the final size of the second phase of the coronavirus epidemic by the logistic model medRxiv 2020.03.11.20024901. doi: https://doi.org/10.1101/2020.03.11.20024901

[36] M. Batista, Estimation of the final size of the COVID-19 epidemic, medRxiv 2020.02.16.20023606. doi: https://doi.org/10.1101/2020.02.16.20023606

[37] M. Merzoukia, M. Bentahirb, M. Najimia, F. Chigra, J-L Gala, The Modeling of the capacity of the Moroccan Health Care System in the context of COVID-19: the relevance of the logistic approach Bulletin of the World Health Organisation (2020), DOI: 10.2471/BLT.20.25868

[38] https://www.cdc.gov/coronavirus/2019-ncov/variants/variant-info.html

[39] https://www.thehindu.com/news/international/who-says-covid-variant-in-india-of-concern/article34529654.ece

[40] https://www.hindustantimes.com/india-news/delta-plus-in-india-40-cases-1st-specimen-found-in-april-sample-wh

[41] https://www.hindustantimes.com/india-news/govt-says-delta-plus-a-variant-of-concern-identifies-3-characteristics

[42] https://www.bbc.com/news/world-asia-india-57564560 